\title{
LGBTIQ-GEFLÜCHTETE ZWISCHEN VULNERABILITÄT UND SELBSTERMÄCHTIGUNG IM ÖSTERREICHISCHEN ASYLSYSTEM
}

Serdar ARshan, Cécile BaLbous, Mag MaCh, Queer Base

\section{INHALT}

1 Einleitung. 143

2 Vulnerabilität (Serdar ARSLAN) ......................................................................... 145

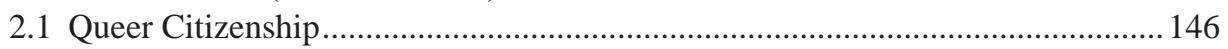

2.2 Vulnerabilität und Prekarität im Asylverfahren ............................................... 148

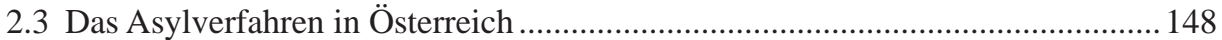

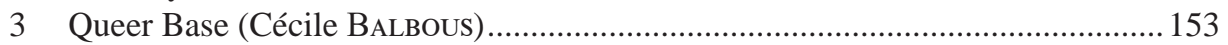

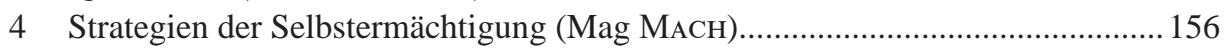

4.1 Gemeinschaftlich gegen Diskriminierung: gegenseitige Unterstützung als

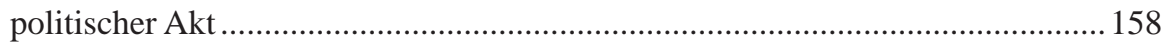

4.2 Queering the Streets: die politische Relevanz der Präsenz von queer-feministischen und intersektionalen Gruppen wie Queer Base bei Demonstrationen ....................................................................................... 160

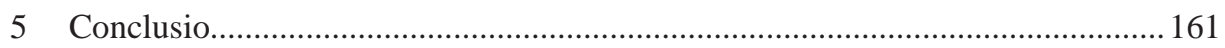

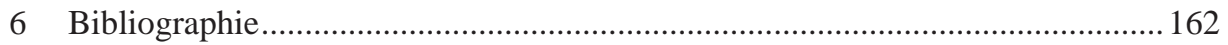

\section{Einleitung}

Im Zuge dieser Arbeit geht es darum, einen genaueren Blick auf die soziopolitische und rechtliche Situation geflüchteter LGBTIQ-Personen und deren Vulnerabili- 
tät im Asylkomplex ${ }^{1}$ in Österreich zu werfen. Diese sind verschiedensten Formen der Diskriminierung ausgesetzt, vor allem dem gesellschaftlichen und institutionellen Rassismus, der Geflüchtete in Österreich und anderen Teilen der Welt insgesamt betrifft. Insbesondere befinden sie sich aber in der schwierigen Lage, dass sie ihre sexuelle Orientierung oder Geschlechtsidentität in einem normativen Rahmen ausführen müssen, der keinen Raum für das Anderssein bietet.

Um diese exkludierenden Mechanismen und normativen Raster auf einer theoretischen, strukturellen Ebene fassen zu können und nicht den Eindruck zu vermitteln, dass es sich dabei um Einzelfälle handelt, beschäftigt sich diese Arbeit zunächst mit den vulnerabilitätstheoretischen Überlegungen Judith BuTLERs (2010). Dabei wird ein Konzept von Vulnerabilität entworfen, welches nicht auf einen situativen Moment beschränkt bleibt, sondern dabei hilft, die Universalisierung eurozentrischer Normen und politischer Strategien im sogenannten Asylkomplex besser zu verstehen. Schließlich drücken sich institutionelle Logiken und Normen tagtäglich in sozialen Praktiken und Entscheidungen aus.

Ebenso wichtig ist es allerdings, die Queer Base und LGBTIQ-Geflüchtete als Akteur/inn/e/n in diesem Feld zu konzipieren, die die Kontingenz von Normen beweisen können. Damit wird nämlich auch deutlich, dass es sich um keine abgeschlossene, lineare Abfolge handelt, sondern eher um einen dialektischen Prozess, in dem Akteur/ inn/e/n in diesem Feld mit staatlichen Institutionen interagieren. Dieses Verständnis ist elementar, um etwaige Veränderungen nachzeichnen und Ausblicke geben zu können.

Im letzten Schritt soll schließlich darauf eingegangen werden, in welchem Verhältnis die Idee eines rassistischen und sexistischen Asylkomplexes zum Thema der Selbstermächtigung LGBTIQ- Geflüchteter steht. Auf welche Weise ist es für Akteur/ inn/e/n wie der Queer Base möglich, eine Infrastruktur zu bieten, die geflüchtete Personen in die Lage versetzt, selbst zu sprechen und handlungsfähig zu werden? Inwiefern können bereits das Ankommen und Überleben in diesen Strukturen als Widerstandsakt Geflüchteter verstanden werden? (vgl. AHMED 2017) Welche (normativen) Kriterien wohnen dem Begriff Widerstand inne? Inwiefern können Solidarität, Sichtbarkeit und gegenseitige Fürsorge auf einer strukturellen Ebene widerständig sein?

Auch wenn bei weitem nicht alle Aspekte des österreichischen Asylkomplexes dargestellt werden können, vermittelt diese Arbeit zum einen eine plausible Idee von normativen Logiken und bietet zum anderen Anknüpfungsmöglichkeiten für weitere Forschung, die den Zusammenhang von Subversivität, Normativität und Vulnerabilität im rechtlichen und alltäglichen Kontext für LGBTIQ-Geflüchtete in Österreich ergründet.

An dieser Stelle wird der Begriff Asylkomplex eingeführt, um eine begriffliche Entsprechung für das hochkomplizierte österreichische Asylsystem zu finden. Zugleich soll durch den Begriff gewährleistet werden, dass verschiedene Machtungleichheiten und normative Raster als ein ineinander verwobenes System begriffen werden, welches dynamisch ist und sich immer wieder in Form rassistischer/sexistischer/trans- und homophober Strategien in die Asylpolitik einschreibt. 


\section{Vulnerabilität (Serdar ARSLAN)}

Dieses Kapitel beschäftigt sich mit der Vulnerabilität und Prekarität geflüchteter, queerer Menschen im Kontext ihres Asylverfahrens in Österreich. Dabei wird zunächst auf das Konzept der Vulnerabilität Judith ButLers eingegangen, um dieses im zweiten Schritt auf die Idee einer „queer citizenship“2 im europäischen Kontext beziehen zu können. Schließlich wird dargestellt, wie sich strukturelle Machtungleichheiten im aktuellen österreichischen Asylkomplex wiederfinden. Die Idee der Vulnerabilität in Bezug auf „queer citizenship“ kann eine theoretische Verknüpfung mit individuell empfundener Ungleichheit im Asylkontext herstellen und damit strukturelle Ungleichheiten verdeutlichen.

Judith BUTLER beschäftigt sich grundsätzlich mit der Frage, warum es möglich ist, dass es Leben gibt, deren Verlust stärker betrauert wird als anderes Leben, dessen Verlust weniger oder manchmal gar nicht problematisiert wird. Gleichsam könnte man sagen, weshalb Leben zunächst bestimmte Kriterien erfüllen oder sich an Normen anpassen muss, um im Asylverfahren das Recht zugesprochen zu erhalten, schützenswert zu sein.

Nach BuTLER gibt es normative Raster, die es ermöglichen, Leben als schützenswert (anzu-)erkennen und anderem Leben die (Schützens-)Wertigkeit abzusprechen bzw. nie zuzusprechen. Demnach kann die Frage nach dem Sein bzw. dem, was als Sein gilt, nicht beantwortet werden, ohne nach den Mechanismen der Macht und gesellschaftlichen Normen zu fragen. Vulnerabilität hingegen ist ein egalitär ausgerichtetes Konzept, das alle Menschen betrifft und sich in ihre Körperlichkeit bereits einschreibt, bevor sie als politisches Subjekt gelten können. Ihre Leiblichkeit wird erst in dem Prozess des von anderen Abhängig- und „Angegangenwordensein(s)“ (PISTROL 2016, p. $235)^{3}$ hergestellt und geformt (vgl. ebd.). Zunächst ist also jeder Mensch vulnerabel, nur wird diese Vulnerabilität oft nicht (an-)erkannt.

Diese grundsätzliche Vulnerabilität bedeutet aber gerade nicht, dass eine gleichverteilte Gefährdung für Menschen besteht, die identitätspolitische Anliegen negiert oder dekonstruiert. Vielmehr soll darauf hingewiesen werden, dass Vulnerabilität stets „unikal“ (PIstrol 2016, p. 259) erfahren wird. Zudem erläutert ButLER, dass in der herrschenden Ordnung die Wahrnehmbarkeit von Leben, die als schützenswert gelten, an normative Raster gebunden ist und die Anerkennung von schützenswertem Leben nur für bestimmte Gruppen möglich ist. Dies bedeutet gleichzeitig nicht, dass es sich um einen einmaligen Akt der Anerkennung handelt oder es einen zentralen Moment des Anerkannt-worden-seins gibt. Vielmehr ist Anerkennung als solche immer in die

2 aus dem Englischen: queere Staatsbürgerschaft.

3 Die Idee des Abhängig- und „Angegangenwordensein(s)“ (PIstrol 2016, p. 235) bezieht sich auf ButLers Idee einer „neue(n) Ontologie des Körpers“ (BuTLER 2010, p. 10).

„Das »Sein« des Körpers, auf welches diese Ontologie verweist, ist ein immer schon anderen überantwortetes Sein, es ist immer schon auf Normen und soziale und politische Organisationen verwiesen (...) Körper sein heißt vielmehr, gesellschaftlichen Gestaltungskräften und Formierungen ausgesetzt sein, weshalb die Ontologie des Körpers immer schon soziale Ontologie ist.“ (BUTLER 2010, p. 10 f.). 
Zukunft gerichtet und muss durch ständige Wiederholung bestätigt werden. Genau in diesem Aspekt besteht für Butler die Veränderbarkeit der Normen (vgl. ButLER 2010, p. 11).

Die Idee einer unterschiedlichen Anerkennbarkeit von Leben ist auch zentral für das Verständnis der Vulnerabilität von LGBTIQ-Personen. Diese wird oftmals erkannt und wahrgenommen, was jedoch nicht dazu führt, dass sie anerkannt wird. Es gibt an dieser Stelle einen elementaren Unterschied zwischen dem Erkennen als Handlung und dem Anerkennen als politischer Strategie. Dem Erkennen folgt also oftmals nicht das Anerkennen, weil die Anerkennung sich auf die bereits erwähnten normativen Raster bezieht. Um Leben als gefährdet wahrnehmen zu können, muss die Gefährdung als „integrale(r) Aspekt” des Lebens erkannt werden und sich „konkretisieren (...) in Fragen wie der Gewährung von Zuflucht, in Fragen der Arbeitsmöglichkeiten, der Ernährung, der medizinischen Versorgung und des entsprechenden Rechtsstatus“ (BUTLER 2010, p. 20). Aber genau das ist in zahlreichen Fällen queerer Geflüchteter nicht der Fall. Und das ist zentral, weil oft erst die Anerkennung eine Handlungsfähigkeit und ein Selbstverhältnis im Sinne des modernen Subjekts ermöglicht (vgl. Pistrol 2016, p. 236). Die fehlende Anerkennung und das Herausfallen aus normativen Rastern führen schließlich dazu, dass LGBTIQ-Geflüchtete gefährdeter sind bzw. prekärer leben als andere Gruppen.

Unterschiedliche Anerkennungsraster sorgen für ihre Diskriminierung und Nichtanerkennung als politisch schützenswerte Subjekte. Hinsichtlich des Begriffs der Prekarität ist zu konstatieren, dass er sowohl die konkrete Situation beschreibt als auch eine Form des Regierens. Denn nach Butler erfordert die Idee einer Sicherheit für manche, die verstärkte Prekarität für andere (vgl. Butler 2012, pp. 7 f.). Das heißt wiederum, dass ein Sicherheitsdiskurs existiert, welcher grundlegende Unsicherheiten für bestimmte Bevölkerungsgruppen oder soziale Gruppen erfordert und produziert. Das zentrale Anliegen dieser prekarisierten und marginalisierten Gruppen kann dann nicht mehr Widerstand und Kritik sein, sondern zunächst die Befreiung aus ihrer maximierten Unsicherheit (vgl. ebd., p. 9).

\subsection{Queer Citizenship}

Ein Ansatz, diesen normativen Druck zu fassen, ist das Konzept der „queer citizenship“. Dieses beschreibt einen Modus der differenzierten Inklusion, die in schützenswertes und nichtschützenswertes Leben unterteilt. Queer citizenship ist als eine Idee zu verstehen, die normativ geäußerte und erkennbare sexuelle Orientierungen und Geschlechtsidentitäten zu einem stereotypisierten Ideal stilisiert, um die oder den idealen queeren Geflüchteten abzubilden und jede Abweichung davon als Unglaubwürdigkeit zu systematisieren (vgl. HARITAWORN et al. 2013, p. 446). (Hetero-)Normative Raster beziehen sich ausdrücklich auch auf queeres Leben und normieren dieses. Auf das Erkennen folgt in diesem Fall auch die Anerkennung, aber eben nur für bestimmte, 
normativen Rastern entsprechende LGBTIQ-Geflüchtete. Demnach geht die Queer Citizenship einher mit einer eng definierten, eurozentrischen, ausschließenden und homonormativen Idee von einer essentialistischen Wahrheit, die LGBTIQ-Personen in irgendeiner Art und Weise verkörpern oder ausdrücken müssen, um als glaubwürdig und damit auch als schutzwürdig anerkannt zu werden. Der Modus der Inklusion von LGBTIQ-Personen ist also normativ und kann nur in einer gewaltsamen Art und Weise erfolgen, die immer auch Ausschlüsse produziert (vgl. ebd., p. 448). Diese Form der Inklusion geht einher mit der Idee von Staatsbürger/inn/e/n als ,self-governing, privatized, and sexually free individual“ (VoLPP 2017, p. 153).

Die Idee eines normativ bestimmbaren, queeren, europäischen Subjekts, das Steuern zahlt, sexuell befreit und integriert ist, hilft zugleich auf einer strukturellen Ebene dabei, eine dichotome Vorstellung von homo-/transphoben „Ländern und toleranten, queerfreundlichen Ländern aus dem globalen Norden zu erzeugen. Diese Inszenierung eines transnationalen, queeren, bestimmbaren Subjekts „,helps directly to foster a model of 'European citizenship' based on the creation of sexual and racial others and simultaneous inclusion of a limited portion of non-heterosexual/non-cisgendered people as part of the citizenry“ (AmmaTuro 2015, p. 1153).

Das Konzept einer europäischen, queeren Citizenship geht einher mit der Vorstellung von guten (queeren) Staatsbürger/inn/e/n, die alle Personen, die sich nicht in dieses Konzept fassen lassen, ausschließt und als „sexual-racial others“ (AмmATURo 2015, p. 1154) identifiziert, die nicht integrierbar sind. Die Verortung des homophoben Moments im „Rest der Welt“ (vgl. Rao 2014) führt zu der paradoxen Situation, dass Länder auf einer diskursiven Ebene als homophob dargestellt werden, aber bei der Bewertung im Asylverfahren nicht homophob genug sind, um die Gefährdung einer aus diesem Land geflüchteten Person anzuerkennen.

"Instead of perceiving all societies as characterized by sexism, homophobia, and transphobia, simply manifested in alternate forms, the world thus becomes organized along a hierarchy of gender and sexual equality development” (VoLPP 2017, p. 170).

Das Moment einer Instrumentalisierung von LGBTIQ-Personen im Kontext einer inszenierten europäischen, queerfreundlichen Staatsbürgerschaft und die Abgrenzung gegen homo-/transphobe Staaten (vgl. Rao 2014) führt zu einer weiteren Prekarisierung und einer verstärkten Prekarität von LGBTIQ- Geflüchteten. Überlegungen im Kontext des Umgangs mit queeren Geflüchteten sind häufig von dieser Dichotomie geprägt. Zum anderen stellt die Idee der Queer Citizenship einen Raster dar, in dem heterosexuelle Beziehungen als Bezugspunkt dienen. Dadurch wird es möglich, eine eurozentrische Vorstellung von Sexualität und normativen Rahmen zu universalisieren und damit die Möglichkeit der Artikulation anderer sexueller Identitäten und Vorstellungen zu verhindern (vgl. HARITAWORN et al. 2013, p. 448). 


\subsection{Vulnerabilität und Prekarität im Asylverfahren}

LGBTIQ-Personen müssen bereits in ihren Herkunftsstaaten mit vielfachen Diskriminierungen und Ausgrenzungen rechnen. Im Falle, dass sie „entdeckt“ werden oder ihre sexuelle Orientierung oder Geschlechtsidentität gegen ihren Willen publik wird, haben sie mit physischer und psychischer Gewalt, Gefängnisstrafen, Kriminalisierung oder sogar dem Tod zu rechnen. Und auch auf der Flucht sind sie oft mit homophoben (Gewalt-)Taten konfrontiert und auf doppelte Weise Gefahren ausgesetzt. Zum einen werden sie, wie die meisten anderen Geflüchteten auch, illegalisiert, diskriminiert, bewusst vergessen oder in Internierungslagern untergebracht. Zugleich sind sie mit stereotypen, diskriminierenden Vorurteilen durch andere Geflüchtete konfrontiert, da sie in keiner Weise Ziel einer besonderen Schutzstrategie sind.

Um diese Probleme und gewaltvoll erlebten Situationen jedoch nicht einseitig im Herkunftsstaat oder auf der sogenannten Reise zu verorten, um damit die Vorstellung des homophoben Globalen Südens zu reproduzieren, sollen im Folgenden die vielfältigen Machtungleichheiten im Asylverfahren im österreichischen Kontext thematisiert werden. Hierzu gibt es zahlreiche Beispiele für individuell erfahrene Diskriminierungen in einem systematisch und institutionell rassistischen und homophoben System, das oben bereits anhand des Konzepts der Queer Citizenship angedeutet wurde.

\subsection{Das Asylverfahren in Österreich}

Grundlage für Entscheidungen bezüglich der Zuerkennung von Asyl sind in Österreich zum einen die Europäische Menschenrechtskonvention (EMRK) und zum anderen die Genfer Flüchtlingskonvention 1951. Letztere definiert eine/n Geflüchtete/n als Person, die aus Gründen

„, [...] der begründeten Furcht vor Verfolgung wegen ihrer Rasse, Religion, Nationalität, Zugehörigkeit zu einer bestimmten sozialen Gruppe oder wegen ihrer politischen Überzeugung sich außerhalb des Landes befindet, dessen Staatsangehörigkeit sie besitzt, und den Schutz dieses Landes nicht in Anspruch nehmen kann oder wegen dieser Befürchtungen nicht in Anspruch nehmen will [...] “ (UNHCR 1967, p. 2).

Dabei gelten in Österreich die sexuelle Orientierung bzw. die Geschlechtsidentität als Merkmale der Zugehörigkeit zu einer sozialen Gruppe. Konkret beginnt das Asylverfahren für LGBTIQ-Personen in Österreich mit dem Erstantrag bei einer Polizeistation. Im Zuge des hierauf folgenden Prozesses ist die asylwerbende Person stets in der Pflicht, ein kohärentes, widerspruchsfreies und plausibles Bild seiner/ihrer sexuellen Orientierung, Geschlechtsidentität und seines/ihres Lebens im Herkunftsstaat und in Österreich darzustellen. Dieses Bild und die Geschichte sind dem ständig wachenden 
Auge der Institutionen ausgesetzt und jeder kleine (angenommene) Widerspruch wird im Folgenden benutzt, um die Glaubwürdigkeit der asylwerbenden Person im Hinblick auf seine/ihre sexuelle Orientierung oder Geschlechtsidentität in Frage zu stellen. Dabei droht, dass nicht nur Widersprüche, sondern auch eine zu detailliert erzählte Geschichte als Unglaubwürdigkeit umgedeutet werden.

Queere Personen sind also im doppelten Sinne der unmöglichen Aufgabe ausgesetzt, ihre Zugehörigkeit zur Gruppe der LGBTIQ glaubwürdig darzustellen. Diese Glaubwürdigkeit wiederum wird anhand verschiedener Fragen ermittelt, die ein inhumanes Eindringen in die Privatsphäre darstellen und gleichzeitig sexuelle Orientierung und Geschlechtsidentität zum essentialistischen Kern einer Identität machen, die sich im Verhalten, Sprechen und Auftreten zu äußern hat. Darauf weist auch Marty HuBER in Ihrem Essay „Radikale Konfliktzone“ hin:

„Dieses Begehren nach Anerkennung durch das Gesetz befördert aber gerade jene essenzialisierenden Mechanismen, die in eine Doktrin der Eindeutigkeit von Geschlecht, normativer (Hetero-) Sexualität und Regierbarkeit eingebettet sind. Es gibt daher stets die Gefahr, dass nur gewisse Ausformungen queeren Lebens als solche anerkannt werden ... " (HuBER 2018, pp. $191 \mathrm{f}$.).

Zugleich betont Sie, dass das Asylverfahren notwendigerweise eine Fähigkeit zum Sprechen voraussetzt - zum Sprechen über Identitäten, Gefühle und intimste Gedanken. Hierbei handelt es sich jedoch nicht um eine Selbstverständlichkeit, vielmehr müsse diese Fähigkeit erst durch die Schaffung emanzipativer, sicherer Räume unterstützt und hergestellt werden (vgl. HuBER 2018, pp. 193 f.).

Das Sprechen in seiner jetzigen Form wird nicht nur vorausgesetzt, sondern sorgt auch dafür, dass die Idee einer jedem Menschen zugehörigen, seine/ihre Identität bestimmenden sexuellen Orientierung oder Geschlechtsidentität immer wieder (re-) produziert wird. Dies führt in der Konsequenz dazu, dass viele Referent/inn/en sich bei Einvernahmen zahlreicher Stereotype bedienen und eine bestimmte Art von Queer-Sein im Sinne der Queer Citizenship erwarten. Da eine Abweichung von diesen Stereotypen als Unglaubwürdigkeit bewertet wird, liegt die Ablehnung zahlreicher Asylanträge auch daran, dass

"(t)he denial (...) is many times related to the "western conception of the linear formation and ultimate fixity of sexual identity', the 'pre-formed expectation of how gay-lesbian or bisexual sexual identity is understood', the judgment based on appearance, and the lack of knowledge about the complexity of the sexuality issue itself, etc." (Pollini 2014, p. 14).

LGBTIQ-Personen in Asylverfahren müssen jedoch nicht nur die Fähigkeit besitzen, ihre Lebensgeschichte erzählen zu können, sondern vor allem die Fähigkeit des linearen Sprechens (über Körperlichkeit und Sexualität) erlernen. In diesem Prozess des Sprechens wird jeder Aspekt der Flucht- und Lebensgeschichte nur zu einem Merkmal der Glaubwürdigkeit degradiert und geflüchtete Menschen werden nicht gehört, son- 
dern objektifiziert und bewertet. Unberücksichtigt bleiben dabei auch die (extremen) Formen der Gewalt, denen diese Menschen ausgesetzt waren bzw. sind. So kommt es zu absurd anmutenden, diskriminierenden Situationen während des Asylverfahrens. Die Tatsache etwa, dass eine Person verheiratet ist und ein Kind hat, beschädigt oftmals die Glaubwürdigkeit des Homosexuell-Seins, wobei Gründe für diese Situation ausgeblendet werden.

Neben diesen offensichtlich unreflektierten und diskriminierenden Mechanismen existiert eine ganze Reihe weiterer Strategien, die die Glaubwürdigkeit einer Person auf andere, subtilere Weisen diskreditiert. All diesen Arten ist allerdings gemeinsam, dass sie sich unreflektiert auf eurozentrische Ideen von Homosexualität, Partnerschaft und Staatsbürgerschaft berufen, wobei jede Widersprüchlichkeit als Unglaubwürdigkeit oder als fehlende Integrationsbereitschaft gewertet wird.

Eine weitere, wichtige Ebene der Beurteilung der Glaubwürdigkeit einer asylwerbenden Person bezieht sich auf das Sprechen über das „öffentliche Ausleben“ der sexuellen Orientierung und geschlechtlichen Identität. Findet diese faktisch nicht statt, so argumentieren Richter/innen und Referent/inn/en meist, dann stelle die sexuelle Orientierung oder Geschlechtsidentität auch im Herkunftsstaat kein Problem dar. Dabei handelt es sich um eine Version des Diskretionsarguments (JANSEN und SPIJKERbOER 2011, p. 33). Hieß es bis vor einigen Jahren noch, eine Diskretion der sexuellen Orientierung stelle in manchen Fällen kein Problem dar, so wird gegenwärtig argumentiert, dass das fehlende öffentliche Ausleben einer sexuellen Orientierung auch nicht geschützt werden muss.

Eine Person ist also nicht schützenswert, wenn sie seine/ihre sexuelle Orientierung oder Geschlechtsidentität nicht auslebt und sei es nur, weil die Person nicht gerne in Bars geht oder Alkohol trinkt. Völlig außer Acht gelassen wird dabei, dass der „,(f) ehlende Kontakt(e) zu anderen Mitgliedern im Aufnahmeland (...) ihre Ursache möglicherweise in wirtschaftlichen Faktoren, der geografischen Lage, der Sprache bzw. kulturellen Barrieren, mangelnder Gelegenheit, persönlichen Entscheidungen oder der Angst vor Bloßstellung“ (UNHCR 2012, p. 29) hat.

Hieran wird deutlich, dass die europäisch normierte, stereotypisierte Idee von Queerness Menschen ausschließt, die verschiedenen Glaubwürdigkeitsmerkmalen nicht entsprechen möchten oder nicht entsprechen können (vgl. Oyewumi 1998). Das zeigt auch, dass das Bild der Richter/innen und Referent/inn/en oftmals total verzerrt ist. Sie verstehen LGBTIQ-Personen als kohärente soziale Gruppe und versuchen die Zugehörigkeit anhand universal gültiger, stereotypisierter Fragen herauszuarbeiten. Dabei sind sie oftmals nicht in der Lage, diese Fragen zu kontextualisieren oder zu reflektieren. Und selbst wenn eine Person sich dahingehend bemüht, Anschluss an eine „queere Szene“ zu finden und aktiv als solche identifizierte Orte besucht, schaffen es Referent/ inn/en, die Glaubwürdigkeit mit anderen Mitteln in Frage zu stellen.

BFA-Einvernahme:

„F: Beschreiben Sie die Regenbogenfahne? 
A: Die Regenbogenfahne steht für die Homosexuellen. Das besteht aus sieben Farben, ich kenne die Farbkombination nicht auswendig.

F: Kennen Sie die Bedeutung einer Farbe?

A: Die Fahne steht für alle, egal ob homosexuell, transsexuell. Ich weiß nicht, welche Farbe symbolisch für was steht. " (BFA-Bescheid - Queer Base-Archiv) ${ }^{4}$

Es gibt zahlreiche Beispiele dafür, dass die Idee der Glaubwürdigkeit an stereotype Vorstellungen gebunden ist, die queere Sexualität normieren und die meisten Facetten derselben eigentlich ausschließen.

“Hence, the act of allowing LGBT persons to 'become'first-class citizens is connected with an attempt to tame their 'diversity', but also to articulate actively their identities to serve concrete political and ideological purposes” (AMMATURo 2015, p. 1154)

Ein weiterer wichtiger Aspekt der Glaubwürdigkeit und Linearität des Sprechens ist das Coming-out. Dieses gilt als einmaliges und abschließbares Moment und nicht als Prozess, der sich stets entwickelt und nicht abgeschlossen ist. Gleichzeitig müsste auch auf das Thema der innerstaatlichen Fluchtalternative eingegangen werden, da diese Option oftmals dann eingesetzt wird, um einer LGBTIQ-Person trotz ihrer Glaubwürdigkeit einen negativen Bescheid ausstellen zu können. Eng mit der innerstaatlichen Fluchtalternative hängt auch die Kategorie der „wohlbegründeten Furcht“ zusammen. Auch hier ist wichtig, dass die asylwerbende Person glaubwürdig nachweist, dass sie bei einer Rückkehr Verfolgung ausgesetzt sein würde. Dabei ist der Diskurs, was genau als Verfolgung zu werten ist, ein ständiger Aushandlungsprozess, der oft zu Ungunsten von Asylwerber/inne/n ausfällt, da die Informationslage nicht ausreichend ist (vgl. PoLLINI 2014, pp. 16 f.). Nicht zuletzt ist die bedrohte Unabhängigkeit der Rechtsberater/innen durch die neue österreichische Bundesagentur für Betreuungs- und Unterstützungsleistungen zu nennen, die insbesondere auch die Arbeit der Queer Base in den nächsten Jahren betreffen wird (Diakonie 2019, pp. 3 f.).

Insgesamt ist also zu konstatieren, dass es sich bei dem Asylkomplex in Österreich um ein rassistisches, sexistisches System handelt, das die Vulnerabilität und Prekarität von asylwerbenden Personen verstärkt oder herstellt, indem sie die Idee des Schutzes von LGBTIQ-Geflüchteten an Normen knüpft und die Zugehörigkeit anhand der Glaubwürdigkeit bewertet. Es ist nicht absehbar, dass sich diese Situation in Zukunft ändern könnte, vielmehr muss davon ausgegangen werden, dass sich der Asylkomplex institutionalisiert hat und einer Logik folgt, die von der österreichischen Regierung bewusst so gesetzt wurde und sich auch unter der neuen Regierung nicht ändern wird (MosAIK BLOG 2019). Damit lässt sich dies als eine Art der Regierung von queeren Geflüchteten beschreiben, die die Unsicherheit und Prekarität dieser Menschen verstärkt und regu-

4 Aufgrund der Tatsache, dass es sich bei den BFA-Bescheiden und BVwG-Erkenntnissen um sensible Daten handelt, deren Veröffentlichung nur unter besonderen Umständen möglich ist, werden an dieser Stelle keine Namen, Daten oder Geschäftszahlen angeführt. Die Zitate können aber durch eine Nachfrage bei der Queer Base bestätigt werden. 
liert, da der rassistische Sicherheitsdiskurs als inklusives Merkmal nur die auch sehr exklusive Form der Queer Citizenship bereithält.

Zugleich ist es wichtig darauf hinzuweisen, dass diese Form des Regierens als generelle Unwilligkeit, geflüchtete Personen aufzunehmen, bewertet werden muss. Zum anderen müssen die Strategien, die im Umgang mit den Prekarisierten vorherrschen, als prozesshaft verstanden werden, denn die Institutionen und die dort arbeitenden Menschen versuchen, bestimmte offensichtlich rassistische oder unreflektierte Fragen zu umgehen, um keine Angriffsfläche zu bieten. Es handelt sich gewissermaßen um einen Organismus, der die Kritik aufnimmt und sie zum Teil verarbeitet, um sich auch auf politischer Ebene abzusichern. So gibt es in der letzten Zeit Richter/innen, die es schaffen, LGBTIQ-Personen zu ihren Gefühlen oder Emotionen zu befragen.

In einem negativen BVwG-Bescheid geht ein Richter auf die UNHCR-Richtlinie aus dem Jahr 2012 ein und unterstreicht die Wichtigkeit von Emotionen. So unterstellt er einem homosexuellen Asylwerber:

„Dem Beschwerdeführer gelang es in diesem Zusammenhang nicht, über seine Empfindungen und Gefühle sowie seine Erfahrungen in Bezug auf Andersartigkeit, Stigmatisierung und Scham substantiiert und glaubwürdig zu berichten. Vielmehr war sein Vorbringen von stereotypen und klischeehaften Aussagen und auf die Durchführung des Geschlechtsverkehrs fokussierten Ausführungen geprägt. “ (BVwG-Erkenntnis, Queer Base Archiv)

Aus diesem Bescheid wird deutlich, dass auch auf institutionell-staatlicher Ebene eine Reaktion auf die Beschwerden und Hinweise durch Vereine wie die Queer Base oder Organisationen wie den UNHCR stattfindet. Auch über die letzten Jahre gesehen gibt es zumindest die Tendenz, dass die Fragen und Bescheide weniger offensichtlich rassistisch oder homophob sind. ${ }^{5}$ Allerdings ist es wichtig zu unterstreichen, dass dies nicht unbedingt dazu führt, dass die Situation für Asylwerber/innen besser geworden ist. Denn auch in dem konkreten Fall schafft es die richtende Person nicht, darüber zu reflektieren, warum die Person nicht über ihre Gefühle spricht (etwa weil sie nicht kann, nicht möchte oder die Frage gar nicht versteht), sondern legt dies als fehlende Glaubwürdigkeit aus (vgl. PoLLINI 2014, pp. 14 f.).

Es ist also wichtig, dass sich die Idee der Glaubwürdigkeit auf andere Bereiche verschiebt, in denen die Diskriminierungen zwar subtiler auftreten, deswegen aber nicht weniger stark. Der Umgang mit Geflüchteten, oder anders gesagt - das Regieren der Prekären und Vulnerablen (durch die Staatsbürgerschaft) - folgt einer rassistischen, homound transphoben, exkludierenden Strategie, deren zentrale Währung die Glaubwürdigkeit und deren zentrales Merkmal das Durchleuchten und Infragestellen dieser ist.

Schlussendlich ist noch darauf hinzuweisen, dass diese Betrachtung der Vulnerabilität im rechtlichen Prozess sich ausschließlich auf die Rechtssituation und die vor-

5 Noch vor zwei Jahren wurde ein Asylgesuch abgelehnt, weil der Asylwerber nicht ,homosexuell wirkte“, vgl. <https://taz.de/Negativer-Asylbescheid-in-Oesterreich/!5528496/>. 
herrschenden Strukturen bezieht. Hierbei droht die Gefahr, LGBTIQ-Geflüchtete einseitig als Betroffene dieser Politik darzustellen, ohne ihnen eigene Handlungsspielräume einzuräumen. Es ist allerdings wichtig, darauf hinzuweisen, dass Butler Vulnerabilität nicht als eine passive Eigenschaft von Menschen konzipiert, sondern vielmehr als aktivistisches Moment bewertet, in dessen Rahmen eine neue Idee der kollektiven Verantwortung und Handlungsfähigkeit entstehen kann, die zunächst der Sichtbarkeit bedarf, um die Kontingenz von Normen herausarbeiten zu können (vgl. PisTrol 2016, pp. 261 ff.). Deshalb geht es in den folgenden beiden Kapiteln darum, auf die Queer Base als Akteurin in diesem Feld einzugehen und anschließend emanzipatorische Möglichkeiten auszuloten, um der Prekarität LGBTIQ-Geflüchteter entgegenwirken zu können.

\section{Queer Base (Cécile Balbous)}

Gegründet im Jahr 2016 ist Queer Base eine Organisation von queeren Menschen mit und ohne Fluchterfahrung in Wien, die geflüchtete Lesben, Schwule, Bisexuelle, Trans- und Intersexpersonen sowie Queers bei ihrem Asylverfahren und danach unterstützt. Dabei geht es aber nicht nur um Fragen des Asylrechts, sondern um komplexe Formen der Auseinandersetzungen mit Fragen der Integration, des Rechtsstaates, fortgesetzter und neuerlicher Homo- und Transphobie, Hoffnungen auf ein besseres Leben etc.

Sie ist die einzige Organisation in Österreich, die Beratung und Unterstützung für LGBTIQ-Geflüchtete anbietet. Anfänglich war das Angebot auf Coming-out-, Sozial-, Rechts- und Grundversorgungsberatung von queeren Geflüchteten im offenen Asylverfahren fokussiert. LGBTIQ- Geflüchtete werden oft jahrelang vom Queer-Base-Team durch das Asylverfahren begleitet, sie sehen in der Queer-Base-Community eine zentrale Stütze und wenden sich auch nach einem positiven Bescheid mit den neuen Herausforderungen an uns. So wurde das Beratungsangebot in Abstimmung mit den Bedürfnissen der Queer-Base-Mitglieder um die Bereiche Wohnen und psychische Gesundheit erweitert. Im Zuge dieser Tätigkeiten haben sich die Themen „Wohnen“ und „Arbeit“ als Problemfelder herauskristallisiert.

In Österreich ist es seit längerer Zeit möglich, unter dem Titel „Angehörige einer sozialen Gruppe“ Asyl aufgrund von homo- bzw. transphober Verfolgung zu beantragen. Jedoch gibt es strukturelle Probleme, die die Situation von LGBTIQ-Geflüchteten, wie im ersten Kapitel beschrieben, verschlechtern. Die Mehrfachdiskriminierung, der LGBTIQ-Geflüchtete ausgesetzt werden, wurde auf internationaler Ebene im ersten Bericht des unabhängigen Experten für den Schutz vor Gewalt und Diskriminierung aufgrund von sexueller Orientierung und Geschlechtsidentität erkannt ${ }^{6}$. So auch

$6 \quad 3^{\text {th }}$ Session of the Human Rights Council; Report of the Independent Expert on protection against violence and discrimination based on sexual orientation and gender identity $\S \S 40,41 \mathrm{~A} /$ HRC/35/36, 19 April 2017. 
in Österreich, wo queere geflüchtete Personen weiterhin von Diskriminierung und Verfolgung seitens der eigenen Community oder von anderen in Österreich lebenden Menschen betroffen sein können. Das Aufsuchen von spezialisierten Beratungsstellen oder ein Coming-out können für viele eine Hürde darstellen. Speziell in ländlichen Gegenden, in denen die Anonymität wie in einer Großstadt und die Erreichbarkeit von Beratungsstellen in geringerem Ausmaß gegeben sind, kann die Situation für Betroffene schwierig sein.

Aufgrund ihrer erhöhten Prekarität und der Diskriminierung in den Flüchtlingsunterkünften sind immer mehr LGBTIQ-Geflüchtete in die TürkisRosaLila Villa, das LGBTIQ-Community Zentrum in Wien, gekommen, einerseits, weil das Haus sichtbar ist und andererseits, weil sie sonst nirgendwo anders hingehen konnten. Am sichtbarsten sind Transgender-Personen, deren Geschlechtsidentität sich abseits der binären Ordnung Mann/Frau verorten lässt. Aus der Notwendigkeit heraus LGBTIQ-Geflüchteten den Zugang zu einem fairen Asylverfahren zu gewährleisten, wurde Queer Base von langjährigen LGBTIQ-Aktivist/inn/en mit und ohne Flucht- oder Migrationserfahrung gegründet.

Das primäre Ziel von Queer Base ist es, sowohl unmittelbarer als auch struktureller geschlechtsbezogener Diskriminierung entgegenzuwirken. Durch Herstellung von Kontakten und den Aufbau eines Community-Netzwerks von LGBTIQ-Akteur/inn/ en sowie durch individuelle Begleitung und Unterstützung von LGBTIQ-Geflüchteten können Gewalt gegen Betroffene und soziale Isolation vermieden werden. Auch kann damit der Zugang zu Gesundheitsversorgung und Bildung erleichtert werden. Durch den Aufbau einer spezialisierten Beratungsstelle mit Schwerpunkt Coming-out und Rechtsberatung sowie Informationsangeboten für Institutionen werden strukturelle und nachhaltige Verbesserungen herbeigeführt. Die Fragen der Rahmenbedingungen im Asylverfahren bzw. des fairen Zugangs zu einem Asylverfahren beinhalten die Frage nach der notwendigen Infrastruktur, um gehört zu werden, um sich artikulieren bzw. äußern zu können wie Gayatri SPIvaK es in ihrem Text „Can the Subaltern Speak?“ (vgl. SPIVAK 2008) formuliert:

"There is no infrastructure for the subaltern to complete their speech act [...] so it's not a question of someone trying literally to listen to muted voices that's a metaphorical description of building infrastructure so that the citizen who is cut off by the structure of the state can actually access them. " 7

In den meisten Flüchtlingsunterkünften müssen LGBTIQ-Geflüchtete ihre sexuelle Orientierung und Geschlechtsidentität aus Sicherheitsgründen verstecken. Ein Fernbleiben von der zugewiesenen Unterkunft zieht einen Verlust der Grundversorgung nach sich und hat Obdachlosigkeit und Mittellosigkeit zur Folge. Die Stadt Wien erkannte dieses Problem im Jahr 2015 und erlaubte Queer Base den Transfer von LGBTIQGeflüchteten in die Grundversorgung Wien und in Kooperation mit dem Projekt Lares

7 Gayatri SPIVAK in einem Interview anlässlich der Kyoto-Prize-Verleihung $2012<$ https:// www.youtube.com/watch? $v=n 8 i P j 6 q k a 3 o>$. 
des Flüchtlingsdiensts Lares und später mit dem Verein Tralalobe die Einrichtung von LGBTIQ- Wohngemeinschaften in Wien. Denn die Entscheidung, in welchem Bundesland eine geflüchtete Person sich während des Asylverfahrens aufzuhalten hat, wird ohne Rücksicht auf die Vulnerabilität der Person vom Innenministerium getroffen.

LGBTIQ-Geflüchtete sind für gewöhnlich in den allgemeinen Grundversorgungseinrichtungen untergebracht und leben daher mit eben jenen Menschen, vor denen sie geflohen sind, zusammen. Dieser Umstand zwingt sie dazu, weiterhin ihren eigentlichen Fluchtgrund zu verstecken. Das Sich-verstecken-müssen, das Befragt-und-inFrage-gestellt-werden, sind die ersten Stufen der Gewalt (vgl. Auger et al. 2008), die in Asylunterkünften stattfinden. Daraus resultieren Mobbing, der Ausschluss aus Community-Netzwerken, mögliche Verdächtigungen und Erpressungsversuche. Ressourcen, Solidarität und Unterstützung durch andere Geflüchtete bleiben aus, weil etwas mit dieser Person ,nicht“ stimmt. Insbesondere durch die prekäre Situation von homosexuellen und Transgender-Asylsuchenden in den Unterbringungen kommt es in Folge zu der Unsicherheit, Opfer von psychischer, physischer und sexualisierter Gewalt zu werden. Erneute mit Homo- und Transphobie verbundene Gewalterfahrungen und Diskriminierungen führen schnell zu Retraumatisierung und schweren Belastungsstörungen.

Es geschieht regelmäßig, dass queere geflüchtete Personen monatelang in völliger Isolation in einer Unterkunft fern der LGBTIQ-Community leben müssen, wo sie sich aus Sicherheitsgründen nicht outen können. Je nach gesellschaftlichen Hintergründen und Erfahrungen mit homo- und transphoben Behörden ist das Coming-out vor der österreichischen Polizei eine der auftretenden Schwierigkeiten. Für manche Geflüchtete, die jahrelang versteckt gelebt haben, sind die Dolmetscher/innen, die aus ihrer Herkunftscommunity stammen, aufgrund der Angst, dass Informationen bezüglich ihrer sexuellen Orientierung und/oder Geschlechtsidentität weitergegeben werden könnten, eine große Herausforderung. Aus Sorge sich durch ein Coming-out zu exponieren bzw. in Gefahr zu bringen, möchten manche Betroffene ihre Geschlechtsidentität und/oder sexuelle Orientierung deshalb verheimlichen. Außerdem haben viele LGBTIQ-Geflüchteten große Schwierigkeiten aufgrund der sexualisierten oder sexuellen Gewalt, des homophoben und transphoben Umfelds, in dem sie leben, der fehlenden (positiven) Sprache zur Selbstbeschreibung über ihre sexuelle Orientierung und Geschlechtsidentität zu sprechen (vgl. UKLGIG 2018).

Queer Base unterstützt sie mit Coming-out-Beratung, mit der Entwicklung einer positiven Sprache in Zusammenarbeit mit den Peer-Dolmetscher/inne/n und mit Workshops zu Selbstermächtigung.

Einige LGBTIQ-Geflüchtete sind schwer von der erlebten Gewalt im Herkunftsland oder von ihrem Fluchtweg traumatisiert und benötigen psychologische, psychotherapeutische bzw. psychiatrische Betreuung. Dazu kommt das Arbeitsverbot für Geflüchtete während des Asylverfahrens, das zu einer Verschlechterung der psychischen Gesundheit führt (vgl. Hentges und StaszczaK 2010). 
Queer Base arbeitet eng mit NGOs und Ärzt/inn/en zusammen, damit LGBTIQGeflüchtete die für sie notwendige medizinische Behandlung erhalten können. Der Verein Queer Base versucht im Rahmen eines Buddy-Programms über Aktivitäten ohne verpflichtende Voranmeldung und Freizeitangebote, die Voraussetzungen für das (Zurück-)Gewinnen einer positiven Zukunftsperspektive für queere geflüchtete Personen zu schaffen, indem die Betroffenen einen positiven Zugang zum eigenen Körper und den eigenen Fähigkeiten (wieder-)erlangen.

LGBTIQ-Geflüchtete dürfen während des Asylverfahrens nicht arbeiten und ihnen wird strukturell die Möglichkeit vorenthalten, ihr Leben aktiv zu gestalten und somit eine optimistische Zukunftsperspektive zu gewinnen. Ein erster Schritt zur Ermöglichung einer positiven Sicht auf die Zukunft ist das Durchbrechen dieser Negativspirale durch (Wieder-)Gewinnung eines positiven Verhältnisses zum eigenen Körper. Die Sportangebote sind „Community-Angebote“, d.h., sie werden auch von LGBTIQ-Geflüchteten mitorganisiert. In der Vergangenheit hat sich gezeigt, dass Schwimm- und Fußballtraining gut für diesen Prozessablauf geeignet sind. So ist auch die Künstler/innen-Gruppe „Squad of Queer Artists“ entstanden und hat an drei Ausstellungen (in Wien und in Salem, Massachusetts, USA) und an der Performance „Queering the Museum“ (Haus 21, Wien) teilgenommen.

Einige Queer-Base-Mitglieder waren vor ihrer Flucht bereits LGBTIQ-Aktivist/ inn/en und setzen mit der Queer Base ihren Kampf fort. Sie vertreten Queer Base in der Öffentlichkeit wie z.B. bei Preisverleihungen wie dem Bruno-Kreisky-Preis oder beim Friedmann-Preis. Sie gestalten den jährlichen Beitrag der Queer Base bei der Regenbogenparade in Wien, und sie halten Reden und Vorträge bei diversen Veranstaltungen. Dadurch werden sie Vorreiter/innen für andere und tragen in dieser Funktion zur Selbstermächtigung queerer geflüchteter Personen bei.

\section{Strategien der Selbstermächtigung (Mag МАCH)}

Im Kontext eines rassistischen und auf Basis von westlichen, queeren Stereotypen operierenden asylpolitischen Systems, welches das Leben geflüchteter queerer und Transpersonen, insbesondere jener mit ausstehendem Asylstatus, bis ins kleinste Detail organisiert und einschränkt, sind eben diese besonders von körperlicher Vulnerabilität und Überwachung im öffentlichen Raum betroffen (vgl. AHMED 2017, p. 238; HaRris und DeFreECE 2016). Strategien zur Selbstermächtigung queerer und Transpersonen im Asylsystem sind daher von nicht zu unterschätzender Wichtigkeit. Strukturelle Gewalt, so wie sie im österreichischen Asylsystem sichtbar wird, ist eine Ansammlung an komplexen und oft versteckten sozialen Prozessen und Praktiken, die ihre Verankerung in Gesetzestexten und Institutionen findet und bestimmte soziale Gruppen strukturell marginalisiert (vgl. El-TAYEB 2009; Mullaly 2010; Ou JIN LeE 2019), was in tiefgehen- 
den und sich über Generationen erstreckenden sozialen Ungleichheiten resultiert. Unter der Kurz-Strache-Regierung (2017-2019) stiegen die Zahlen rassistischer Gewalt in Österreich laut des Zivilcourage \& Anti-Rassismus-Arbeit-Netzwerks (ZARA) an (vgl. Schennach und SchreiLeChner 2019). Durch offen diskriminierende Sprache in der Flüchtlingspolitik und restriktive Auslegung der ohnehin schon sehr restriktiven Gesetzestexte unter dieser rechten Regierung erhielten die strukturell diskriminierenden Praktiken öffentlicher Institutionen das grüne Licht aus höchster Riege. Boulevardzeitschriften heizten das soziopolitische Klima durch ausländerfeindliche Artikel an, wodurch ein gesellschaftlicher Rechtsruck weiter gefördert wurde. Dies zeigt ein Schema auf, das von G.W. Smith folgendermaßen analysiert wurde:

“... ruling regimes can be described as a complex set of text-mediated processes and practices which flow through multiple sites of power, including political and bureaucratic apparatuses, social institutions, and media" (OU JIN LEE 2019, p. 74).

Forschung zu struktureller Gewalt hat gezeigt, dass Betroffene in Reaktion auf solche Gewalt stets, je nach Umständen offen oder versteckt, Wege des Widerstands finden (vgl. KineweSQuAO und Wade 2009, p. 205). Eine Reaktion oder Antwort auf institutionelle Diskriminierung bzw. strukturelle Gewalt von Seiten betroffener Personen kann somit als Widerstandsakt bezeichnet werden. Überleben allein - in einem System, das auf dem Ausschluss des „Anderen“ beruht - kann Protest sein (vgl. AHMED 2017, p. 237). In ihrem Gedicht „A Litany for Survival“, das jenen gewidmet ist, die „nie überleben sollten“, thematisiert Audre Lorde dieses Überleben als politischen Akt:

"For those of us

who were imprinted with fear

like a faint line in the centre of our foreheads

learning to be afraid with our mother's milk

for by this weapon

this illusion of some safety to be found

the heavy-footed hoped to silence us

For all of us

this instant and this triumph

We were never meant to survive.” (LORDE 1978, p. 31)

In diesem Abschnitt werden wir spezifisch darauf eingehen, wie queere geflüchtete Personen innerhalb ihrer vom Staat sehr eingeschränkten Situation gemeinschaftlich Widerstandsstrategien finden können, sich in bestimmten Bereichen zu emanzipieren und inwiefern community-basierte Organisationen, wie Queer Base, dazu beitragen können. 


\subsection{Gemeinschaftlich gegen Diskriminierung: gegenseitige Unterstïtzung als politischer Akt}

Queer Base und ähnliche gemeinschaftsorientierte und von queeren, geflüchteten Personen mitgeführte Organisationen bieten einen essentiell wichtigen Raum für Gemeinschaft, eine Art Rettungsschnur, zu der queere und Transpersonen im für sie extrem isolierenden Asylsystem sonst kaum Zugang haben. Sie bietet einen sicheren Raum, in dem gegenseitige Unterstützung durch gleichgesinnte Personen mit ähnlichen Lebenserfahrungen zu finden ist. Wie man sich um sich und um andere sorgt und einander gegenseitig unterstützt in einem Kontext, in dem man aufgrund von Sexualität und Geschlechtsidentität, sozialer Herkunft, Hautfarbe, Religionszugehörigkeit und Staatsbürgerschaft marginalisiert wird, ist ein politischer Akt feministischer Fürsorge ${ }^{8}$ (vgl. Ahmed 2017, p. 237). Diesem Prinzip entsprechend bietet die Community, die sich in und um die community-basierte Organisation Queer Base gebildet hat, eine Plattform und einen Rückzugsort für Formen dieser politisch relevanten queer-feministischen Fürsorge und gegenseitiger Unterstützung. Formen und Strategien sich gegen strukturelle Diskriminierung und Benachteiligung in der österreichischen Gesellschaft und im Asylsystem aufzulehnen und einander darin gegenseitig zu unterstützen, die in der Queer-Base-Community praktiziert bzw. angeboten werden, sind neben der Rechtsund Sozialberatung, auch die Wohnbuddy-Gruppe, wöchentliche Treffen wie das Queer Café oder gemeinschaftliche Feiern und Mental-Health-Treffen.

In der Wohnbuddy-Gruppe beispielsweise helfen Freiwillige, die Erfahrung mit dem Wiener Wohnungsmarkt haben, queeren und Transpersonen, die eben ihren Asylbescheid bekommen haben, eine passende Wohnung oder ein WG-Zimmer zu finden. Nach der Zustellung des Asylbescheides hat die betroffene Person vier Monate Zeit, um einen Wohnplatz außerhalb des Asylsystems zu suchen. Innerhalb dieser Zeitspanne muss die Person zunächst eine bezahlte Arbeit finden, da von Vermieter/inne/n auf dem offiziellen Wohnungsmarkt durchwegs ein Einkommensnachweis verlangt wird. Ohne Einkommensnachweis kann daher nur auf dem inoffiziellen Wohnungsmarkt, d.h. nach WG-Zimmern, gesucht werden. Für die Aufgabe, innerhalb der 4-monatigen Übergangsphase einen Wohnplatz zu finden, ist daher eine Unterstützung, wie sie die Wohnbuddy-Gruppe bietet, unentbehrlich. Die dringende Notwendigkeit der Unterstützung ist für viele jedoch nur deshalb gegeben, weil das System sie in eine Lage bringt, in der es für sie unmöglich ist, diese Herausforderungen alleine zu bewältigen. Strukturell ist der Wohnungsmarkt insofern für Personen, die gerade einen Asylbescheid erhalten haben, kaum zugänglich, da die meisten Vermieter/inn/en einen Einkommensbescheid mit einem Gehalt in der dreifachen Höhe der Monatsmiete verlangen und jemanden, der Mindestsicherung bezieht, als Mieter/in nicht akzeptieren würden. Es mangelt zumeist auch an finanziellen Mitteln, um die Kaution und die erste Monatsmiete zu bezahlen, sowie auch am Wissen darum, wie der Wiener Wohnungs- und Arbeitsmarkt funktio-

8 Im Original als „feminist care“ bezeichnet. 
niert. Die Beratung, das gemeinsame Suchen und die Unterstützung bei der Beantragung des Kautionsgeldes beim Sozialamt der MA 40 stellen daher im Rahmen der Hilfe zur Verselbständigung der Personen mit positivem Asylbescheid grundlegende Aufgaben der Wohnbuddies dar.

Des Weiteren ist die Begleitung durch einen Buddy für die Wohnungsbesichtigung strategisch wichtig, da Vermieter/innen zuweilen offen oder versteckt diskriminierend ihre Auswahl treffen. So werden beispielsweise Anfragen von Personen mit ausländischen Namen, besonders betrifft dies Namen arabischer Herkunft, mit deutlicher Skepsis behandelt oder nicht beantwortet. Bei der Besichtigung spielt die Person, die als Wohnbuddy fungiert, somit eine wichtige Vermittlerrolle, um rassistische Diskriminierungsformen zumindest temporär bzw. teilweise umgehen zu können. Die Aufgabe der Wohnbuddy-Gruppe besteht also darin, jenen mit Beratung und Begleitung zur Seite zu stehen, die sich im Kontext der strukturellen Benachteiligung von Asylberechtigten auf dem Wohnungs- und Arbeitsmarkt in Österreich von der Betreuung im Asylsystem emanzipieren wollen bzw. die dabei Hilfe benötigen.

Gemeinschaftliches Beisammensein anlässlich der wöchentlichen Treffen, wie das Queer Café, gemeinschaftliche Feiern oder auch das Mental-Health-Treffen haben den Zweck Räume zu schaffen, in denen sich queere und Transpersonen mit Fluchterfahrung untereinander austauschen können und sich willkommen fühlen ohne sich für ihre intersektionalen Identitäten erklären zu müssen. Es geht darum, ein Gefühl der Gemeinschaft und des Zusammenhalts aufzubauen sowie einen Ort der Zugehörigkeit und des solidarischen Miteinanders zu schaffen. Dieses solidarische Miteinander allein ist schon ein „politischer Akt feministischer Fürsorge“ (vgl. Ahmed 2017, p. 237). Die Last des Navigierens im öffentlichen Raum und auch in sozialen Kontexten im vorrangig weißen, cis/hetero geprägten Österreich, die der Besitz einer oder multipler marginalisierter Identitäten mit sich bringt (vgl. HARRIS und DEFrEeCE 2016), wird im gemeinschaftlichen Zusammensein, das einen lokalen „queer \& of colour“-Minikosmos aufbaut, aufgehoben bzw. zu einem wesentlichen Teil verringert. Dies stellt somit eine unentbehrliche feministische Praxis dar, die einen Rückzugsort und Raum für gemeinschaftliche Fürsorge schafft. In diesem Kontext des gemeinschaftlichen Vertrauens und Zusammenhalts können in Folge sensible Themen wie Fluchterfahrungen, Formen der Vergangenheitsbewältigung und Diskriminierungserfahrungen in der österreichischen Gesellschaft zur Sprache kommen. Dies macht die Gemeinschaft, die die Queer-BaseCommunity bietet, zu einem unentbehrlichen und einzigartigen Bestandteil des österreichischen queeren Sozialgefüges. 


\subsection{Queering the Streets: die politische Relevanz der Präsenz von queer-feministischen und intersektionalen Gruppen wie Queer Base bei Demonstrationen}

Im vorrangig weißen cis/hetero und christlich-konservativen soziopolitischen Kontext in Österreich ist die Repräsentation von Personen, die nicht weiß, cis/hetero und/oder im Besitz einer österreichischen Staatsbürgerschaft sind, eine Rarität. Strukturelle sozialpolitische Kräfte halten die tiefsitzenden neokolonialen Machtverhältnisse und sozialen Ungleichheiten (vgl. Foucault 1978; Harris und Defreece 2016), die eine Marginalisierung der als „anders“ Stigmatisierten voraussetzen, am Laufen. Ein Ausdruck dessen sind die Ausschlusspraktiken, denen Körper von multiplen, marginalisierten Identitäten im öffentlichen Raum ausgesetzt sind - hauptsächlich ausgeübt und/ oder implizit unterstützt von jenen, die privilegierter im sozialen Gefüge sind, inklusive jenen mit queerer Identität (vgl. Harris und Defreece 2016, p. 136; Puar 2007). Daher ist es essentiell für queere und Transpersonen mit Fluchterfahrung, nicht nur Zugang zu einer solidarischen Gemeinschaft zu haben, die sie in ihren intersektionalen Identitäten widerspiegelt, sondern auch zu Möglichkeiten sich selbst im öffentlichen Raum repräsentiert zu sehen. Da diese in der österreichischen Gesellschaft noch kaum gegeben sind, liegt es zumeist an der Community selbst diese Repräsentation, eine Selbstrepräsentation also, im öffentlichen Raum zu schaffen: beispielsweise im gemeinschaftlichen Auftreten und Teilnehmen an Demonstrationen. Dies ist die politische Relevanz und symbolische Wirkkraft der Präsenz dieser Gemeinschaft im öffentlichen Raum und politischen Diskurs, wie auf den „wieder-do“-Demonstrationen, die im Frühling 2019 zur Absetzung der Kurz-Strache-Regierung beigetragen haben. Die gemeinschaftliche Präsenz dieser Community auf der Straße allein ist schon ein politisches Statement. Gerade da Personen mit multiplen marginalisierten Identitäten, die außerhalb der österreichischen, weißen, cis/hetero, christlich-konservativen Norm liegen, einer viel höheren körperlichen Prekarität und Überwachung im öffentlichen Raum ausgesetzt sind, ist dieser Schritt gemeinschaftlich auf die Straße zu gehen nicht nur umso politischer, sondern birgt vor allem auch ein viel größeres Risiko für eine ohnehin schon prekarisierte Gruppe. Im Falle von Zusammenstößen mit Gegendemonstrant/inn/en oder der Polizei sind Angehörige marginalisierter Minderheiten mit einer viel höheren Wahrscheinlichkeit Beschimpfungen, physischer Gewalt und Verhaftungen ausgesetzt. Durch diese Prekarität von nichtweißen, queeren und Transpersonen mit Fluchterfahrung sind ein intersektional-feministischer Ansatz und die Präsenz von österreichischen queeren und Trans-Aktivist/inn/en im kollektiven Auftreten als Queer-Base-Community in Demonstrationen von Bedeutung, um den Forderungen derjenigen Personen Gehör zu verschaffen, deren Stimmen strukturell ungehört verhallen, und um gleichzeitig als Puffer zu Gegendemonstrant/inn/en oder zur Polizei zu fungieren. 


\section{Conclusio}

"Each of us must find our work and do it. Militancy no longer means guns at high noon, if it ever did. It means actively working for change, sometimes in the absence of any surety that change is coming" (LORDE 1982).

Da die Arbeit der Queer Base diesem Prinzip folgt, wurde auch in diesem Beitrag die soziopolitische (und rechtliche) Situation geflüchteter LGBTIQ-Personen und insbesondere deren Vulnerabilität im Kontext des Asylkomplexes in Österreich von einer von aktivistischer Praxis ausgehenden Seite aus beleuchtet.

Die einleitende Darlegung vulnerabilitätstheoretischer Überlegungen Judith BuTLERS (2010) erklärte die Zusammenhänge zwischen der Universalisierung eurozentrischer Normen (Ammaturo 2015; Fassin 2010; Oyewumi 1998) und politischer Strategien im sogenannten Asylkomplex im österreichischen Kontext. So wurden von einer kritischen Analyse der institutionellen Ebene ausgehend jene strukturellen Diskriminierungsformen und Ausschlussmechanismen erfasst, die queere geflüchtete Personen in Österreich betreffen. In Beispielen wurde dargelegt, wie sich diese institutionellen Logiken und Normen im Alltag betroffener Personen, im Besonderen in Asylbescheidsentscheidungen, ausdrücken. Im Folgenden wurde die Rolle der lokalen NGO Queer Base beschrieben und erörtert, inwiefern sich queere geflüchtete Personen und Aktivist/ inn/en im Kampf gegen diese strukturellen Diskriminierungsformen organisieren und engagieren. Im Widerstand gegen strukturell tief verankerte Ausschlussmechanismen in einem rassistischen Asylsystem (vgl. EL-TAYEB 2009), das auf dem Ziel aufbaut möglichst viele Personen nicht aufnehmen zu müssen, ist es von großer Wichtigkeit, dass marginalisierte Bevölkerungsgruppen wie queere geflüchtete Personen innerhalb der Zivilgesellschaft Wege finden können sich selbst zu organisieren.

„Die Grenzen schützen!” ist ein rechter Wahlspruch, der seit 2015 an politischem Gewicht gewonnen hat und nun auch unter der neuen türkis-grünen Regierung einer der wichtigsten Programmpunkte ist (MosAIK-BLOG 2019). Während der Klimaschutz durch diverse Maßnahmen gefördert wird - mit dem Ziel Österreich bis 2040 klimaneutral zu machen - fehlt jedoch jede Verbindung zwischen der Klimapolitik mit sozialen Fragen und die rechtslastige Asylpolitik der Kurz-Strache-Regierung (2017-2019) wird mit neuer Wirkkraft von dieser Regierung weitergeführt. So wird, wie im ersten Teil umrissen, durch die Errichtung der Bundesagentur für Betreuungs- und Unterstützungsleistungen (BBU) ab Ende dieses Jahres ein geschlossenes Asylsystem geschaffen, in das die Zivilgesellschaft kaum noch Einblick hat. Im Kontext dieser politischen Entwicklungen, die schwerwiegende Konsequenzen für queere geflüchtete Personen haben werden, die künftig in Österreich um Asyl ansuchen, ist es von umso größerer Relevanz, dass die Zivilgesellschaft und insbesondere auch Gruppen, die von queeren geflüchteten Personen und Menschen mit multiplen marginalisierten Identitäten mitorganisiert werden, sich für die Rechte marginalisierter Bevölkerungssegmente noch deutlicher engagieren und Selbstermächtigungsstrategien fördern. 


\section{Bibliographie}

Ahmed S. (2017), Living a Feminist Life. Durham, Duke University Press.

Ammaturo F.R. (2015), The 'Pink Agenda': Questioning and Challenging European Homonationalist Sexual Citizenship, Sociology, 49 (6), pp. 1151-1166.

Ammaturo F.R. (2017), European Sexual Citizenship - Human Rights, Bodies and Identities. London, University of Roehampton.

Amnesty International (2014), The Human Cost of Fortress Europe: Human Rights Violations against Migrants and Refugees at Europe's Borders. [Report] London, UK.

Auger N., Fracchiolla B., Moïse C. \& Schultz-Romain C. (2008), De la violence verbale, pour une sociolinguistique des discours et des interactions. In: Durand J., Habert B. \& LaKs B. (eds.), Congrès Mondial de Linguistique Française, pp. 631-643.

Bakshi, S., Jivraj, S. \& Posocco, S. (2016), Decolonizing Sexualities: Transnational Perspectives Critical Interventions. Oxford: Counterpress.

Bundesamt Für Fremdenwesen und Asyl (ed.) (2018), Bescheid. Queer Base-Archiv.

ButLer J. (2010), Raster des Krieges. Warum wir nicht jedes Leid beklagen. Frankfurt/New York, Campus Verlag.

Butler J. (2012), Vorwort. In: Lorey, I. (ed.), Die Regierung der Prekären. Wien, Verlag Turia + Kant, pp. 7-12.

Bundesverwaltungsgericht (ed.) (2019), Erkenntnis: Queer Base-Archiv.

Cruz A. \& Manalansan M.F. (2002), Queer Globalizations: Citizenship and the Afterlife of Colonialism. New York, London, New York University Press.

Diakonie Österreich (ed.) (2019), Stellungnahme der Diakonie Österreich zum Bundesgesetz, mit dem die Bundesagentur für Betreuungs- und Unterstützungsleistungen Gesellschaft mit beschränkter Haftung errichtet und das BFA-Verfahrensgesetz, das Asylgesetz 2005 und das Grundversorgungsgesetz - Bund 2005 geändert werden (BBU-Errichtungsgesetz$B B U-G)$. Report [online] Vienna, Austria.

El-Tayeb F. (2009), The Birth of a European Public: Migration, Postnationality and Race in the Uniting of Europe. In: GutiÉrrez D.G. and Hondagneu-Sotelo P. (eds.), Nation and Migration: Past and Future. Baltimore, Johns Hopkins University Press, pp. 649-70.

FASSIN É. (2010), National identities and transnational intimacies: Sexual democracy and the politics of immigration in Europe, Public Culture, 22 (3), pp. 507-529.

FAssin É. (2012), Sexual democracy and the new racialization of Europe, Journal of Civil Society, 8 (3), pp. 285-288.

Ferreira N. (2018), Reforming the Common European Asylum System: Enough Rainbow for Queer Asylum Seekers?, GenIUS, 2, pp. 25-42.

Foucault, M. (1978), The History of Sexuality: An Introduction, vol. 1. New York, Vintage.

European Union Agency for Fundamental Rights (FRA) (2018), Being Black in the EU: Second European Union Minorities and Discrimination Survey. Luxembourg: Publications Office of the European Union [online].

Haritaworn J., Kuntsman A. \& Posocco S. (2013), Murderous inclusions, International Feminist Journal of Politics, 15 (4), pp. 445-452.

Harris L. \& Defreece A.W. (2016), Body control: The neocolonial displacement of the multiple minority queer body in urban space, Humanity \& Society, 40 (2), pp. 133-154. 
Hentges G. \& StaszczaK J. (2010), Geduldet, nicht erwünscht. Auswirkungen der Bleiberechtsregelung auf die Lebenssituation geduldeter Flüchtlinge in Deutschland, An Interdisciplinary Series of the Centre for Intercultural and European Studies - CINTEUS, 6 (hg. von V. Hinnenkamp, A. Honer, G. Hentges \& H. W. Platzer). Stuttgart, Ibidem Verlag.

Huber M. (2018), Radikale Konfliktzone: Sexuelle Minderheiten auf der Flucht. In: KüPPERS C. \& Schneider M. (eds.), Orte der Begegnung. Orte des Widerstands. Zur Geschichte homosexueller, trans/-geschlechtlicher und queerer Räume. Männerschwarm Verlag, pp. 190-198.

Jansen S. \& Spijkeboer T. (2011), Fleeing Homophobia - Asylum Claims Related to Sexual Orientation and Gender Identity in Europe. [Report] Amsterdam, Vrije Universiteit Amsterdam.

KinewesQuao [Richardson C.] \& WAde A. (2009), Taking resistance seriously: A responsebased approach to social work in cases of violence against Indigenous women. In: STREGA, S. \& Esquao, S.A. (eds.), Walking this Path Together: Anti-racist and Anti-oppressive Child Welfare. Winnipeg, MB, Fernwood, pp. 204-220.

Lorde A. (1978), A litany for survival. In: Collected Poems of Audre Lorde. NYC, W. W. Norton \& Company, Inc.

LORDE A. (1982), Learning from the 60s. In: Sister Outsider: Essays and Speech. Berkeley, CA, Crossing Press, pp. 134-44.

Moore H.K.V. (2018), Lessons from LGBTIQ refugee-led community-based organisations, $F M R, 58$, pp. 67-69.

Mosaik-Blog (2019), Regierungsprogramm. Grüner Anstrich für ’s türkise „,weiter so “ [online] $<$ https://mosaik-blog.at/regierungsprogramm-tuerkis-gruen-analyse/ $>$.

Mullaly B. (2010), Challenging Oppression and Confronting Privilege ( $2^{\text {nd }}$ ed.). Don Mills, ON, Oxford University Press.

Ou Jin LeE, E. (2019), Responses to Structural Violence: The Everyday Ways in Which Queer and Trans Migrants with Precarious Status Respond to and Resist The Canadian Immigration Regime, International Journal of Child, Youth and Family Studies, 10 (1), pp. 70-94.

OYewumi O. (1998), De-confounding gender: Feminist theorizing and Western culture, a comment on Hawkesworth's confounding gender, Signs, 23 (4), pp. 1049-1062.

PISTROL F. (2016), Vulnerabilität. Erläuterungen zu einem Schlüsselbegriff im Denken Judith Butlers, Zeitschrift für Praktische Philosophie, 3 (1), pp. 233-272.

PolLini F.E. (2014), The difficult path towards the recognition of refugee status based on sexual orientation and gender identity, ICL Journal, pp. 1-51.

PuAR J.K. (2007), Terrorist Assemblages: Homonationalism in Queer Times. Durham, NC, Duke University Press.

RAo, R. (2014), The locations of homophobia, London Review of International Law, 2 (2), pp. 169-199.

Schennach P., Schreilechner A.-L. (ed.) (2019), Rassismus Report 2018: Einzelfall-Bericht über rassistische Überfälle und Strukturen in Österreich. Wien, Zivilcourage \& AntiRassismus Arbeit (ZARA).

SPIVAK G.C. (2008), Can the Subaltern Speak? Postkolonalität und subalterne Artikulation. Wien, Turia+Kant (im Orig.: Can the Subaltern Speak, 1988).

UK Lesbian \& Gay Immigration Group (ed.) (UKLGIG) (2018), Still Falling Short. Report [online]. 
UNHCR (ed.) (1967), Abkommen über die Rechtsstellung der Flüchtlinge vom 28. Juli 1951. Protokoll über die die Rechtsstellung der Flüchtlinge vom 31. Januar 1967 [online].

UNHCR (2010), Summary Conclusions: Asylum-Seekers and Refugees Seeking Protection on Account of their Sexual Orientation and Gender Identity [online].

UNHCR (2012), Richtlinien zum Internationalen Schutz, Nr. 9. [online].

VolpP L. (2017), Feminist, Sexual, and Queer Citizenship. In: SHACHAR, A., Bauböck, R., BloemraAd, I., \& Vink, M. (eds.), The Oxford Handbook of Citizenship. Oxford University Press, pp. 153-186. 